\title{
Aportación de la ecografía realizada por enfermería a la exploración del acceso vascular
}

\author{
Patricia Molina Mejías, Beatriz Liébana Pamos, Yolanda Moreno Pérez, Patricia Arribas Cobo, Pilar \\ Rodríguez Gayán, Pilar Díaz de Argote
}

Hospital Universitario Infanta Leonor. Madrid. España

\section{Resumen}

En los últimos años, ha comenzado a usarse por parte de enfermería la ecografía vascular en las unidades de diálisis, mejorando el conocimiento del acceso vascular (AV), ayudando en la elección de las zonas de canulación y aumentando la confianza en los AV nuevos o dificultosos.

Nuestro objetivo es describir los motivos de exploración del AV con ecógrafo, los hallazgos encontrados y contrastarlos con el servicio de radiología.

Es un estudio observacional, descriptivo en 22 pacientes con fistula arteriovenosa (FAV). Diseñamos un registro donde se recogieron los datos de las exploraciones ecográficas y se explotaron con el programa SPSS15.

Se realizaron 37 exploraciones en 22 pacientes. Las principales causas de la exploración fueron: dificultad o dolor en las punciones, localización de nuevas zonas de punción, extravasaciones previas, vigilancia en la maduración de la FAV y seguimiento de FAV. Hallamos $35.1 \%$ con trombos, $8.1 \%$ circulación colateral, $5.4 \%$ colecciones/hematomas y $13.5 \%$ estenosis. De los 22 pacientes, 6 se derivaron a radiología y se contrastaron 2 estenosis, 1 disminución de flujo y 1 trombo.

Las exploraciones ecográficas realizadas por enfermería de forma autónoma se identifican disfunciones del AV aportándonos mayor información sobre el AV.

PALABRAS CLAVE: ecografía; enfermería; fístula arteriovenosa; acceso vascular; hemodiálisis.

Correspondencia:

Patricia Molina Mejías

Servicio de Nefrología del Hospital Universitario

Infanta Leonor. Madrid

C/Zazuar, $9,3^{\circ}$ C. 28031 Madrid

E-mail: ey_frey_7@hotmail.com
Contribution of the ultrasound performed by nursing to the exploration of vascular access

\begin{abstract}
In recent years, vascular ultrasound has been used in nursing dialysis units, improving knowledge of vascular access (VA), helping in the selection of cannulation zones and increasing confidence in new or difficult VAs.
\end{abstract}

Our objective is to describe the reasons for the exploration of VA with ultrasound, the findings found and contrast them with the radiology service.

It is an observational, descriptive study in 22 patients with arteriovenous fistula (AVF). We designed a graph where data from ultrasound scans were collected and exploited with SPSS15.

The sample consisted of 22 patients. There were 37 explorations. The main causes of the exploration were mainly difficulty or pain in the punctures followed by the localization of new areas of puncture, previous extravasations, surveillance in the maturation of AVF and follow-up of AVF. We found $35.1 \%$ with thrombi, $8.1 \%$ collateral circulation, $5.4 \%$ collections / hematomas and $13.5 \%$ stenosis. Of the 22 patients, 6 were referred to radiology and 2 stenosis, 1 decrease of flow and 1 thrombus were tested.

A high number of ultrasound examinations by nursing are carried out autonomously with which AV dysfunctions are identified, contributing more information about AV.

KEYWORDS: ultrasound; nursing; AVF; hemodialysis. 


\section{Introducción}

Para obtener un acceso arteriovenoso válido que pueda utilizarse para iniciar programa de hemodiálisis (HD) crónica hay que sortear numerosos obstáculos. El mayor de todos ellos es conseguir una adecuada maduración. Una vez alcanzado este difícil objetivo, hay que seguir en estado de alerta y utilizar todos los medios disponibles a nuestro alcance para evitar la trombosis y mantener la permeabilidad del acceso vascular (AV).

La trombosis irreversible del AV ocasiona una serie de consecuencias negativas para el paciente que sigue programa de HD: reducción del capital venoso, necesidad de la implantación de un catéter venoso central (CVC), menor eficacia de la HD, posible estenosis o trombosis de venas centrales, inflamación crónica en el caso de las FAVp, y la construcción de un nuevo AV. Todo ello incrementa la frecuencia de hospitalización, la morbimortalidad y el gasto sanitario del paciente en HD crónica. Por tanto, evitar la trombosis del AV es primordial para estos pacientes.

Según las últimas guías de acceso vascular recién editadas, se recomienda que las unidades de hemodiálisis dispongan de programas protocolizados de seguimiento del AV con participación multidisciplinar. Estos programas deben incluir métodos para detectar precozmente la disfunción del AV, localizar su origen y efectuar el tratamiento electivo.

Estos programas de seguimiento deben desarrollarse en cada unidad de HD de forma sistemática, protocolizada y con una participación multidisciplinar de enfermería, nefrología, radiología y cirugía vascular.

La ecografía es una técnica de imagen que permite la exploración, tanto de las FAV nativas como de las FAV protésicas, mediante un transductor lineal emisor y receptor de ultrasonidos aplicado en los distintos planos del AV. Es un método de diagnóstico rápido que puede ser utilizado in situ en la sala de $H D$, así como un método reglado de vigilancia para la evaluación periódica del $A V$, aporta información hemodinámica del $\mathrm{AV}$, es una técnica de elección para confirmar, localizar y cuantificar la estenosis del AV detectada mediante los métodos de cribado antes de efectuar el tratamiento electivo y permite la valoración morfológica y funcional de otras disfunciones del $\mathrm{AV}$ no relacionadas con la estenosis o trombosis como aneurismas y pseudoaneurismas, hematomas, abscesos, etc ${ }^{1}$.
La enfermería, debidamente formada, está capacitada para realizar exploraciones ecográficas, facilita un diagnóstico precoz de las complicaciones por lo que permite participar en la toma de decisiones del $A V$ en la propia sala de hemodiálisis ${ }^{2-3}$.

La ecografía se ha convertido en una herramienta fundamental ya que es el único método no invasivo que nos aporta información anatómica y hemodinámica al mismo tiempo. De este modo, en los últimos años se ha ido implementando la ecografía en las unidades de diálisis y cada vez más por personal de enfermería. El uso del ecógrafo por enfermería mejora el conocimiento del $\mathrm{AV}$, ayuda en la elección de las zonas de canulación y aumenta la confianza de enfermería en los AV nuevos o dificultosos ${ }^{4-5}$.

Desde hace tres años se ha fomentado la formación del personal de enfermería de nuestra unidad en uso y manejo del ecógrafo para exploración del acceso vascular. Desde entonces, seis enfermeras han realizado formación específica, realizando posteriormente talleres de formación para el resto del personal de la unidad y desde hace dos años contamos con un ecógrafo en nuestra unidad.

Para ver como esa formación ha repercutido en las exploraciones realizadas, se decide poner en marcha este trabajo y poder valorar el grado de consolidación del uso del ecógrafo por el personal de enfermería.

Por este motivo, nos planteamos conocer cuántas exploraciones de fístulas arteriovenosas se han realizado mediante ecografía por parte de enfermería en un servicio de nefrología. Siendo los objetivos secundarios: describir los motivos de exploración del AV con ecógrafo, describir los hallazgos encontrados, comparar la fiabilidad de los hallazgos ecográficos con los radiológicos y determinar los factores de riesgo que están relacionados con la aparición de complicaciones detectables mediante ecografía.

\section{Material y Método}

Se realizó un estudio observacional, descriptivo realizado en pacientes portadores de FAV en programa de hemodiálisis crónica y prediálisis del centro de diálisis del Hospital Universitario Infanta Leonor en el periodo comprendido entre octubre de 2016 y febrero de 2017.

Los criterios de inclusión fueron: pacientes portadores de FAV de nuestra unidad de diálisis, mayores de 18 
años que, a criterio del personal de enfermería de la sala de diálisis, se decidiera realizar exploración ecográfica y que hubieran dado su consentimiento informado.

Se incluyeron a 22 pacientes en los cuales se realizaron 37 exploraciones lo que supuso nuestra muestra de estudio.

Las ecografías fueron realizadas por personal de enfermería formado previamente y se realizaron en la misma sala de diálisis en los pacientes en programa de HD 0 en la consulta de enfermería de Enfermedad Renal Crónica Avanzada (ERCA) en el caso de los pacientes con FAV en periodo de maduración que no estaban en programa de HD.

La formación se realizó de la siguiente manera; 6 enfermeras acudieron a un curso presencial teórico práctico de ecografía del acceso vascular para enfermería que prepara a los alumnos para realizar un reconocimiento anatómico de la $\mathrm{FAV}$, detección de parámetros de maduración y funcionamiento, realizar medición del flujo de la FAV, localización de puntos de punción y punción ecoguiada. Posteriormente se realizaron tres talleres teórico prácticos presenciales en la nuestra unidad dirigidos por el personal ya formado y apoyo de otros profesionales, así como de personal de la casa comercial del ecógrafo portátil de la unidad para la instrucción del resto del personal, consiguiendo de este modo que un elevado porcentaje de profesionales de enfermería estuvieran capacitados para realizar exploraciones ecográficas del $\mathrm{AV}$.

A todos los pacientes se les explicó en que iba a consistir el estudio y todos nos dieron su consentimiento de forma verbal.

Todas las exploraciones se realizaron con el ecógrafo portátil Sonosite M-Turbo ${ }^{\circledR}$ que disponemos desde enero de 2015 y sobre el cual se recibió la formación de los talleres presenciales de la unidad.

La exploración consistía en exploración física previa de la FAV (observación, palpación y auscultación) para detectar alteraciones del soplo, thrill o anatómicas (hematomas, aneurismas, signos de infección...) y exploración ecográfica con valoración anatómica de vasos, anastomosis, medición datos como calibres, profundidades y flujo de acceso, si se precisaba para valorar zonas de punción, detectar presencia de trombos, estenosis y presencia de flujo.
Para llevar a cabo este estudio diseñamos una gráfica de recogida de las variables del estudio (figura 1 ), que se completaba cada vez que realizábamos alguna exploración ecográfica a nuestros pacientes.

Se recogieron parámetros demográficos: la edad y el sexo, y parámetros relativos al AV: tiempo que llevaba realizada la FAV, tipo de FAV (autóloga o protésica) y la localización de la FAV.

Respecto a la exploración las variables recogidas fueron: causas del estudio ecográfico, exploración física previa del AV y hallazgos encontrados con el ecógrafo y derivación a estudio radiológico.

Posteriormente, en el caso de aquellas que fueron derivadas al servicio de Radiología, se recogieron de las historias clínicas, los informes de las exploraciones realizadas por este servicio con el que se contrastaron nuestros datos.

Tratamiento estadístico: El análisis estadístico se realizó con el programa SPSS 15.0. Las variables cualitativas se muestran con porcentajes. El test chi-cuadrado fue utilizado para contrastar las variables cualitativas.

Un valor de $p$ menor de 0.05 fue considerado como estadísticamente significativo.

\section{Resultados}

Se realizaron 37 exploraciones en 22 pacientes, de los cuales $13(59.1 \%)$ eran hombres y el $9 \%(40.9 \%)$ eran mujeres. La edad media era de 69.72 años.

Se identificaron 20 (90.9\%) FAV nativas y 2 (9.7\%) FAV protésicas. De ellas, 26 (70.3\%) eran FAV humerocefálicas, $8(21.6 \%)$ eran radiocefálicas y $3(8.1 \%)$ eran humeroaxilares.

Las causas por las que se realizó la exploración ecográfica fue en un $32.4 \%$ por la dificultad o dolor en las punciones, en un $16.2 \%$ para la localización de nuevas zonas de punción, en un $10.8 \%$ para valorar extravasaciones previas, en un $8.1 \%$ para vigilar maduración y evolución de la FAV y un $8.1 \%$ por seguimiento de la FAV.

En la exploración previa a la valoración ecográfica, la observación mostraba que un $27 \%$ de las FAV presentaban un aspecto normal, que un $43.2 \%$ tenían aneurismas, un $18.9 \%$ presentaban hematomas y un $8.1 \%$ tenía edemas. De la palpación, podemos decir que un 
$91.9 \%$ de las FAV tenían thrill normal y un $8.1 \%$ no tenían thrill o era débil. En la auscultación, un $86.5 \%$ presentaban soplo normal y un $13.5 \%$ tenían ausencia de soplo, débil o piante. De las 37 exploraciones, en 1 de ellas había ausencia de flujo.

Entre los hallazgos encontrados tras realizar la ecografía encontramos en un $35.1 \%$ presencia de trombos, en un $8.1 \%$ circulación colateral, en un $5.4 \%$ había colecciones/hematomas y en un $13.5 \%$ había estenosis.

De los 22 pacientes, 6 de ellos se derivaron al servicio de radiología. De los 3 que tenían dificultad en la canalización uno de ellos presentó una estenosis y las otros dos FAV eran profundas; y en las otras 3, derivadas por disfunción, se encontró 1 estenosis, 1 disminución de flujo y 1 trombo.

Contrastando los datos que obtuvimos en la observación de las FAV con los hallazgos encontrados en la exploración ecográfica, encontramos que existe una relación estadísticamente significativa entre la presencia de trombos y las FAV aneurismáticas $(p=0.003)$.

No encontramos relación estadísticamente significativa entre la presencia de estenosis o trombosis, el tipo de FAV y el sexo del paciente.

\section{Discusión}

En nuestra unidad, desde que empezó la formación del personal, se realizan un alto número de exploraciones ecográficas por parte del personal de enfermería de forma autónoma previamente a la elaboración de este trabajo, por lo que el personal de enfermería cada vez ha presentado mayor confianza y autonomía en su uso habitual. Se realizan principalmente por tener dificultad en las punciones, por disfunción del acceso, para realizar las primeras punciones tras la maduración de la FAVn o en algunas ocasiones para localizar nuevas zonas de punción. Estas ecografías nos aportan mayor información sobre nuestros accesos que, en algunas ocasiones, se contrasta con posterioridad con el servicio de Radiología, principalmente cuando se trata de patología que pudiera precisar intervencionismo.

La principal causa por la que se realizan las exploraciones ecográficas en nuestro estudio ha sido las dificultades en la canalización y el dolor en la punción, seguida de la localización de nuevas zonas de punción, como ya se ha descrito en varios trabajos de enfermería, lo que demues- tra el auge que está teniendo la ecografía en la ayuda para realizar las punciones ecodirigidas ${ }^{3-6}$.

La ecografía por el personal de enfermería nos facilita el detectar patología que dificulta el funcionamiento de la FAV, también facilita la canulación en caso de FAV dificultosa, así como la valoración de la maduración previo a la primera punción, como ya se ha demostrado en estudios previos ${ }^{1-7-8-9}$ y como ha sido nuestro caso.

Consideramos que los hallazgos encontrados por enfermería tienen una elevada fiabilidad dado que la mayoría de ellos se pueden contrastar con lo hallado en radiología, en los casos en los que se ha realizado posteriormente. Por ello, al igual que en otros estudios se afirma, el uso del ecógrafo por enfermería debe utilizarse con orientación diagnóstica y de toma de decisiones².

Al existir en nuestro estudio una relación estadísticamente significativa entre la presencia de trombos y las FAVs aneurismáticas, reafirmando lo indicado en guías clínicas, podemos aconsejar que se debe evitar la punción en estas área, puesto que ayuda a la aparición de aneurismas en la zona de punción y podría favorecer el desarrollo de trombos en el AV.

Podemos concluir que se realizan un alto número de exploraciones ecográficas por enfermería de forma autónoma, con las que se identifican disfunciones del AV aportándonos información relevante sobre el mismo.

Se requiere de futuros estudios para ampliar el tamaño muestra y poder realizar un análisis más profundo de los datos.

\section{Agradecimientos}

Queremos agradecer ante todo el esfuerzo realizado y la implicación e interés de todos nuestros compañeros de la Unidad de Hemodiálisis del Hospital Infanta Leonor quienes realizaron las ecografías a nuestros pacientes y recogieron todos los datos de nuestro estudio ecografía tras ecografía.principalmente cuando se trata de patología que pudiera precisar intervencionismo. 


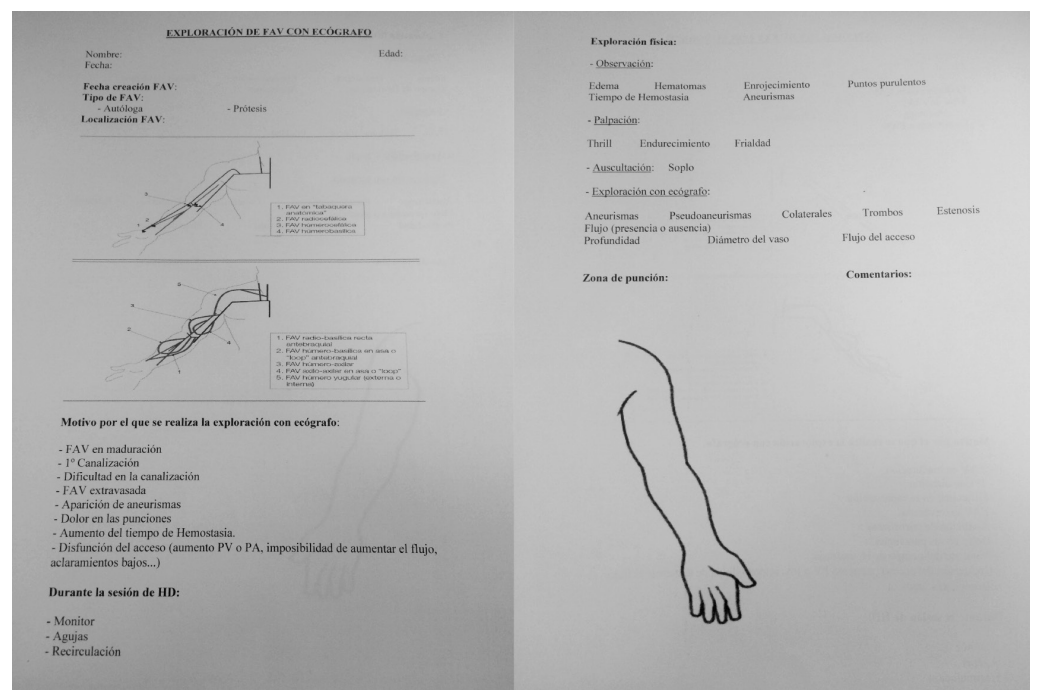

Figura 1. Gráfica de recogida de datos de la exploración ecográfica del AV.

Recibido: 21 abril 2017

Revisado: 27 mayo 2017

Modificado: 8 junio 2017

Aceptado: 21 junios 2017

\section{Bibliografía}

1. Sánchez A, Villoria S, Muñoz B, Sánchez ML, Turrión V. El ecógrafo como herramienta de trabajo de enfermería en hemodiálisis extrahospitalaria. Enferm Nefrol 2014; 17 Suppl (1):116-140.

2. Vinuesa X, Iglesias R, Moya MC, Vallespin J, Fortuño JR, Ibeas JA. Formación, validación y evaluación de un equipo de enfermería nefrológica en el uso de ecografía del acceso vascular para hemodiálisis. Enferm Nefrol 2016; 19 Suppl (1): 49-55.

3. Rueda L, Martín M, Galiano FR. Eco-doppler como herramienta en la punción de fistulas arteriovenosas. Enferm Nefrol 2015; 18 Suppl (1): 101137.

4. Roca-Tey R, Ibeas J, Moreno T, Gruss E, Merino JL, Vallespín J, Hernán D, Arribas P. Monitorizazión y vigilancia del acceso vascular. En: Guía española de acceso vascular para Hemodiálisis. Grupo Español Multidisciplinar de Acceso Vascular (GEMAV). Capítulo 4. [Consultado 7 marzo 2017]. Disponible en: http://www.senefro.org/modules.php?name $=$ webstructure $\& a m p ; i d w e b s t r u c t u r e=418$.

5. Rueda L, Martín M, Galiano FR, Gómez AS. Abordaje de fistulas arterio-venosas bajo visión ecográfica. Enferm Nefrol 2015; 18 Suppl (1): 101-137.

6. López L, Fernández C, Díaz L, Ornosa C. Uso de la ecografía como apoyo de la técnica de redireccionamiento de la aguja en el dolor de la fíaistula arteriovenosa durante la hemodiálisis. Enferm Nefrol 2015 enero-marzo; 18 (1): 58/60.

7. Garrido V, Sesmero C, Ortigosa A, Gruss E. Valoración de la implantación del seguimiento ecográfico del acceso vascular autólogo. Enferm Nefrol 2015; 18 (4).

8. Valle M, Sesmero C, Ortigosa A, Gruss E. Valoración de la implantación del seguimiento ecográfico del acceso vascular autólogo en la unidad de nefrología. Enferm Nefrol 2015; Octubre-Diciembre; 18 (4): 260-264.

9. Sánchez A, Villoria $S$, Muñoz B, Sánchez ML, Turrión V, El ecógrafo como herramienta de trabajo de enfermería en hemodiálisis extrahospitalaria. Enferm Nefrol 2014; 17 Suppl (1): 116-140.

10. Fontseré N,Caro P, MartíA, Roca-Tey R, Ibeas J,Pozo JL, Arribas P, Martínez MT. Cuidados de la fístula arteriovenosa. En: Guía española de acceso vascular para Hemodiálisis. Grupo Español Multidisciplinar de Acceso Vascular (GEMAV). Capítulo 3. [Consultado 7 marzo 2017]. Disponible en: http://www. senefro.org $/$ modules. php? name=webstructure \&amp;idwebstructure $=418$. 